\title{
The number of closed ideals in $L\left(L_{p}\right)$
}

\author{
WiLliam B. Johnson \\ Texas A\&M University \\ College Station, TX, U.S.A.
}

by

\author{
Gideon Schechtman \\ The Weizmann Institute of Science \\ Rehovot, Israel
}

\section{Introduction}

For a reasonably complete discussion of the history of constructing closed ideals in $L\left(L_{p}\right)$, see the introduction in [JPS]. Here, we just remark that in 1981, Bourgain, Rosenthal, and the second author [BRS] constructed $\aleph_{1}$ mutually non-isomorphic complemented subspaces of $L_{p}:=L_{p}(0,1)$ for $1<p \neq 2<\infty$, thereby producing (as noted in $[\mathrm{P}]$ ) $\aleph_{1}$ different closed ideals in $L\left(L_{p}\right)$. (It is of course well known that the compact operators are the only closed ideal in $L\left(L_{2}\right)$.) At that time it was open whether, absent the continuum hypothesis, $L\left(L_{p}\right)$ contains a continuum of closed ideals. Recently, Schlumprecht and Zsák [SZ] built a continuum of closed ideals in $L_{p}:=L_{p}(0,1)$.

The main contribution of this paper is Theorem 4.1, in which we prove that $L\left(L_{p}\right)$, $1<p \neq 2<\infty$, has exactly $2^{2^{\aleph_{0}}}$ different closed ideals.

Recall the notions of small and large closed ideal in $L(X)$. An ideal is called small if it is contained in the ideal of strictly singular operators. Otherwise it is called large. The ideals built in [SZ] are all small, while the ones coming from infinite-dimensional complemented subspaces are clearly large. Our basic construction is designed to produce large ideals. Note that there are at most a continuum of mutually non-isomorphic complemented subspaces of $L_{p}$ (as the density character of $L\left(L_{p}\right)$ and of the set of projections on $L_{p}$ is the continuum). So necessarily we produce different kinds of ideals. Unfortunately, we do not produce any new complemented subspaces of $L_{p}$.

The new large ideals in $L\left(L_{p}\right)$ that we construct are "smallish" in the sense that, even though there are idempotents in the ideals whose ranges are isomorphic to $\ell_{2}$ (see Remark 4.2), no operator in any of the ideals is an isomorphism on a copy of $\ell_{p}$. The Kadec-Pełczyński dichotomy principle $[\mathrm{KP}]$ implies that every complemented subspace

W. B. J. was supported in part by NSF DMS-1900612. G. S. was supported in part by Israel Science Foundation. 
of $L_{p}$ that is not isomorphic to a Hilbert space contains a complemented subspace that is isomorphic to $\ell_{p}$. Consequently, the range of any infinite-rank idempotent in any of the ideals that we construct in Theorem 4.1 (and, as we said, there are infinite-rank idempotents in the ideals) must be isomorphic to $\ell_{2}$.

To put these new "smallish" large ideals into perspective within the Banach algebra $L\left(L_{p}\right)$, notice that it follows from the Kadec-Pełczyński dichotomy principle [KP] that there are exactly two different minimal large closed ideals in $L\left(L_{p}\right)$ when $2<p<\infty$, and thus also for $1<p<2$ (because an operator $T$ in $L\left(L_{p}\right)$ is strictly singular if and only if $T^{*}$ is strictly singular on $L_{q}, 1 / p+1 / q=1$, by Weis' theorem [W]). The first of these is $\Gamma_{\ell_{p}}\left(L_{p}\right)$, the ideal of operators that factor through $\ell_{p}$. This ideal is closed because an operator $T: X \rightarrow L_{p}, 2<p<\infty$, factors through $\ell_{p}$ if and only if $I_{p, 2} T$ is compact, where $I_{p, 2}$ is the formal identity mapping from $L_{p}$ into $L_{2}$; see [J]. One can prove using the Kadec-Pełczyński dichotomy principle $[\mathrm{KP}]$ that $I_{p, 2} S$ is compact whenever $S$ is a strictly singular operator on $L_{p}$, so the alternate characterization of $\Gamma_{\ell_{p}}\left(L_{p}\right)$ for $2<p<\infty$ also yields that $\Gamma_{\ell_{p}}\left(L_{p}\right)$ contains all strictly singular operators on $L_{p}, 2<p<\infty$, and thus also for $1<p<2$ by [W].

The second minimal large closed ideal in $L\left(L_{p}\right)$ is the closure $\bar{\Gamma}_{2}\left(L_{p}\right)$ of the ideal $\Gamma_{2}\left(L_{p}\right)$ of operators on $L_{p}$ that factor through a Hilbert space. Here the closure is needed; in fact, it is not hard to see that there are compact operators on $L_{p}$ that do not factor through a Hilbert space.

We recall in passing that, as was noted in [JPS], the situation in $L\left(L_{1}\right)$ is nicer: $\Gamma_{\ell_{1}}\left(L_{1}\right)$ is the unique minimal closed large ideal in $L\left(L_{1}\right)$ and it contains all the strictly singular operators on $L_{1}$.

In Remark 4.3 we prove that the new large ideals we construct in $L\left(L_{p}\right)$ do not contain the strictly singular operators on $L_{p}$, and hence neither does $\bar{\Gamma}_{2}\left(L_{p}\right)$. All previously known large ideals in $L\left(L_{p}\right)$ other than $\bar{\Gamma}_{2}\left(L_{p}\right)$ do contain the strictly singular operators, and this is the first proof that $\bar{\Gamma}_{2}\left(L_{p}\right)$ does not. A byproduct of Remark 4.3, stated as Remark 4.4, is that $L\left(L_{p}\right)$ contains exactly $2^{2^{\aleph_{0}}}$ small closed ideals.

Our construction and proof of Theorem 4.1 consist of two steps. In $\S 2$ we state and prove the technical Proposition 2.1. This easily yields Corollary 2.3, which gives a general criterion for a space with an unconditional basis to contain $2^{2^{\aleph_{0}}}$ different closed ideals. The criterion is in term of the existence of a special operator on the space.

In $\S 3$ we show that, for $1<q<2$, the space $L_{q}$ contains a complemented subspace (this is Rosenthal's $\mathfrak{X}_{q}$ space, which has an unconditional basis) that admits an operator satisfying the criterion of Proposition 2.1. The construction here borrows a lot from a previous similar construction from [JS]. Duality and complementation then imply the main result. 


\section{The main proposition}

There is a continuum of infinite subsets of the natural numbers $\mathbb{N}$, each two of which have only finite intersection. Denote some fixed such continuum by $\mathcal{C}$. For a finite-dimensional normed space $E$, we denote by $d(E)$ the Banach-Mazur distance (isomorphism constant) of $E$ to a Euclidean space. Also, recall that, for an operator $T: X \rightarrow Y$ between two normed spaces, $\gamma_{2}(T)$ denotes its factorization constant through a Hilbert space:

$$
\gamma_{2}(T)=\inf \{\|A\|\|B\|: T=A B, A: H \rightarrow Y, B: X \rightarrow H, H \text { a Hilbert space }\} .
$$

If $T$ is of rank $k$, then $\gamma_{2}(T) \leqslant k^{1 / 2}\|T\|$ because every $k$-dimensional normed space is $k^{1 / 2}$-isomorphic to $\ell_{2}^{k}$ [T-J, Theorem 15.5]. Note that $d(E)$ is just $\gamma_{2}\left(I_{E}\right)$, where $I_{E}$ is the identity operator on $E$.

Proposition 2.1. Let $X$ be a Banach space with a 1-unconditional basis $\left\{e_{i}\right\}$, let $Y$ be a Banach space, and let $T: X \rightarrow Y$ be an operator of norm at most 1 satisfying the following conditions:

(a) For some $\eta>0$ and for every $M$, there is a finite-dimensional subspace $E$ of $X$ such that $d(E)>M$ and $\|T x\|>\eta\|x\|$ for all $x \in E$.

(b) For some constant $\Gamma$ and every $m$, there is an $n$ such that every $m$-dimensional subspace $E$ of $\left[e_{i}\right]_{i \geqslant n}$ satisfies $\gamma_{2}\left(\left.T\right|_{E}\right) \leqslant \Gamma$.

Then, there exist natural numbers $1=p_{1}<q_{1}<p_{2}<q_{2}<\ldots$ such that, denoting

$$
G_{k}:=\left[e_{i}\right]_{i=p_{k}}^{q_{k}}
$$

for each $k$, defining, for each $\alpha \in \mathcal{C}$, the operator $P_{\alpha}: X \rightarrow\left[G_{k}\right]_{k \in \alpha}$ to be the the natural basis projection, and setting $T_{\alpha}:=T P_{\alpha}$, the following statement holds: If $\alpha_{1}, \ldots, \alpha_{s} \in \mathcal{C}$ (possibly with repetitions) and $\alpha \in \mathcal{C} \backslash\left\{\alpha_{1}, \ldots, \alpha_{s}\right\}$ then, for all $A_{1}, \ldots, A_{s} \in L(Y)$ and all $B_{1}, \ldots, B_{s} \in L(X)$,

$$
\left\|T_{\alpha}-\sum_{i=1}^{s} A_{i} T_{\alpha_{i}} B_{i}\right\| \geqslant \frac{\eta}{2}
$$

Proof. Note first that we can strengthen condition (a) to include also that, given any $n$, one can chose the subspace $E$ to also satisfy that it is contained in $\left[e_{i}\right]_{i>n}$. Now choose inductively $1=p_{1}<q_{1}<p_{2}<q_{2} \ldots$ so that, for each $k, G_{k}=\left[e_{i}\right]_{i=p_{k}}^{q_{k}}$ contains a subspace $E_{k}$ with $\|T x\|>\eta\|x\|$ for all $x \in E_{k}$ and

$$
d\left(E_{k}\right) \geqslant q_{k-1}
$$

(as we will see, it is enough that $d\left(E_{k}\right) / q_{k-1}^{1 / 2} \rightarrow \infty$ ) and, if $E$ is a subspace of $H_{k}=$ $\left[G_{l}\right]_{l=p_{k+1}}^{\infty}$ with $\operatorname{dim} E \leqslant q_{k}$, then

$$
\gamma_{2}\left(\left.T\right|_{E}\right)<\Gamma .
$$


Let now $P_{\alpha}: X \rightarrow\left[G_{k}\right]_{k \in \alpha}$ be the natural basis projection and set $T_{\alpha}:=T P_{\alpha}$.

Suppose that $\alpha_{1}, \ldots, \alpha_{s} \in \mathcal{C}$ (possibly with repetitions) and $\alpha \in \mathcal{C} \backslash\left\{\alpha_{1}, \ldots, \alpha_{s}\right\}$. Assume to the contrary that there are $A_{1}, \ldots, A_{s} \in L(Y)$ and $B_{1}, \ldots, B_{s} \in L(X)$ such that

$$
\left\|T_{\alpha}-\sum_{i=1}^{s} A_{i} T_{\alpha_{i}} B_{i}\right\|<\frac{\eta}{2}
$$

There are infinitely many $k \in \alpha \backslash \bigcup_{i=1}^{s} \alpha_{i}$. For each such $k$, let $R_{k}$ be the basis projection onto $\left[G_{l}\right]_{l<k}$ and $Q_{k}$ the basis projection onto $\left[G_{l}\right]_{l>k}$. Now, for any $i=1, \ldots, s$, we have $T_{\alpha_{i}} G_{k}=0$ since $k \notin \alpha_{i}$, and $\operatorname{dim}\left(R_{k} B_{i} E_{k}\right) \leqslant q_{k-1}$ and $\operatorname{dim}\left(B_{i} E_{k}\right) \leqslant q_{k}$, so we get that, for each $i$,

$$
\begin{aligned}
\gamma_{2}\left(\left.A_{i} T_{\alpha_{i}} B_{i}\right|_{E_{k}}\right) & \leqslant \gamma_{2}\left(\left.A_{i} T_{\alpha_{i}} R_{k} B_{i}\right|_{E_{k}}\right)+\gamma_{2}\left(\left.A_{i} T_{\alpha_{i}} Q_{k} B_{i}\right|_{E_{k}}\right) \\
& \leqslant q_{k-1}^{1 / 2}\left\|A_{i}\right\|\left\|B_{i}\right\|+\Gamma\left\|A_{i}\right\|\left\|B_{i}\right\| .
\end{aligned}
$$

Consequently,

$$
\gamma_{2}\left(\left.\sum_{i=1}^{s} A_{i} T_{\alpha_{i}} B_{i}\right|_{E_{k}}\right) \leqslant\left(\max _{i=1}^{s}\left\|A_{i}\right\|\left\|B_{i}\right\|\right) s\left(q_{k-1}^{1 / 2}+\Gamma\right) .
$$

On the other hand, since $\|x\| \geqslant\left\|T_{\alpha} x\right\| \geqslant \eta\|x\|$ for all $x \in E_{k},(2.2)$ implies that

$$
\left(1+\frac{\eta}{2}\right)\|x\| \geqslant\left\|\sum_{i=1}^{s} A_{i} T_{\alpha_{i}} B_{i} x\right\| \geqslant \frac{\eta\|x\|}{2}
$$

for all $x \in E_{k}$. Since $d\left(E_{k}\right) \geqslant q_{k-1}$, we deduce that

$$
\gamma_{2}\left(\left.\sum_{i=1}^{s} A_{i} T_{\alpha_{i}} B_{i}\right|_{E_{k}}\right) \geqslant \frac{\eta}{2+\eta} q_{k-1} .
$$

For $k$ large enough, this contradicts (2.3).

Remark 2.2. Observe that the only condition on $T_{\alpha}$ that was used to get the inequality (2.1) is that $\|x\| \geqslant\left\|T_{\alpha} x\right\| \geqslant \eta\|x\|$ for all $x$ in $E_{k}$ with $k \in \alpha$. Consequently, the proof of Corollary 2.3 below shows that any operator $S$ in $L(X)$ for which there is $\eta>0$ such that $\|S x\| \geqslant \eta\|x\|$ for all $x$ in $E_{k}$ with $k \in \alpha$ cannot be in the closed ideal generated by $\left\{T_{\beta}: \beta \in \mathcal{C}\right.$ with $\left.\beta \neq \alpha\right\}$. In fact, from the proof of Proposition 2.1, only the inequality $\|S x\| \geqslant \eta\|x\|$ for all $x$ in $H_{k}$ with $k \in \alpha$ and where $H_{k}$ is isomorphic to $E_{k}$ with isomorphism constant independent of $k$ is sufficient to conclude that $S$ is not in the closed ideal generated by $\left\{T_{\beta}: \beta \in \mathcal{C}\right.$, with $\left.\beta \neq \alpha\right\}$. This observation will be used in Remark 4.3 at the end of this paper. 
Corollary 2.3. Let $X$ be a Banach space with a 1-unconditional basis $\left\{e_{i}\right\}_{i}$ and assume there is an operator $T: X \rightarrow X$ of norm at most 1 satisfying (a) and (b) of Proposition 2.1. Then, $L(X)$ has exactly $2^{2^{\aleph_{0}}}$ different closed ideals.

Proof. For any non-empty proper subset $\mathcal{A}$ of $\mathcal{C}$, let $\mathcal{I}_{\mathcal{A}}$ be the ideal generated by $\left\{T_{\alpha}\right\}_{\alpha \in \mathcal{A}}$; i.e., all operators of the form $\sum_{i=1}^{s} A_{i} T_{\alpha_{i}} B_{i}$ with $s \in \mathbb{N}, A_{i}, B_{i} \in L(X), \alpha_{i} \in \mathcal{A}$, $i=1, \ldots, s$. To avoid cumbersome notation, interpret $\mathcal{A} \subset \mathcal{C}$ to mean that $\mathcal{A}$ is a non-empty proper subset of $\mathcal{C}$.

Since we allow repetition of the $T_{\alpha_{i}}$, it is easy to see that this really defines a (nonclosed) ideal. Let $\mathcal{B}$ be a subset of $\mathcal{C}$ different from $\mathcal{A}$ and assume, without loss of generality, that $\mathcal{B} \not \subset \mathcal{A}$. Let $\alpha \in \mathcal{B} \backslash \mathcal{A}$. Then, by Proposition 2.1, $T_{\alpha} \notin \overline{\mathcal{I}}_{\mathcal{A}}$. Consequently, $\left\{\overline{\mathcal{I}}_{\mathcal{A}}\right\}_{\mathcal{A} \subset \mathcal{C}}$ are all different.

Since the density character of $L(X)$, for any separable $X$, is at most the continuum, it is easy to see that, for any separable space $X, L(X)$ has at most $2^{2^{\aleph_{0}}}$ different closed ideals.

Remarks 2.4. (1) One can strengthen the conclusion of the corollary by getting an antichain of $2^{2^{\aleph_{0}}}$ closed ideals in $L(X)$; i.e., such a collection no two of whose members are included one in the other. For that one just uses a collection of $2^{2^{\aleph_{0}}}$ subsets of $\mathcal{C}$ no two of which are included one in the other.

(2) Similarly, one gets a collection of $2^{\aleph_{0}}$ different closed ideals in $L(X)$ that form a chain (by taking a chain of subsets of $\mathcal{C}$ of that cardinality). It is also easy to show by a density argument that, for any separable $X$, this is the maximal cardinality of any chain of closed ideals in $L(X)$.

(3) If $Y$ is a Banach space that contains a complemented subspace $X$ with the properties of Corollary 2.3, then clearly $L(Y)$ also has $2^{2^{\aleph_{0}}}$ different closed ideals (actually an antichain). The same is true also for any space isomorphic to such a $Y$.

(4) The simplest examples of spaces $X$ that satisfy the hypotheses of Corollary 2.3 and thus $L(X)$ has $2^{2^{\aleph_{0}}}$ different closed ideals are $\left(\sum_{i} \ell_{r_{i}}^{n_{i}}\right)_{2}$ for $r_{i} \uparrow 2$ and $n_{i}$ satisfying $n_{i}^{1 / r_{i}-1 / 2} \rightarrow \infty$. It is not hard to show that the identity operator on such a space satisfies (a) and (b) of Proposition 2.1. Consequently, by (3), $L\left(\left(\sum_{i} \ell_{r_{i}}\right)_{2}\right)$ for $r_{i} \uparrow 2$ also has $2^{2^{\aleph_{0}}}$ different closed ideals. Interesting, but less natural, examples of separable spaces $X$ with $L(X)$ having $2^{2^{\aleph_{0}}}$ different closed ideals were known before (see [M]). Unfortunately, $\left(\sum_{i} \ell_{r_{i}}^{n_{i}}\right)_{2}$ for $r_{i} \uparrow 2$ and $n_{i}^{1 / r_{i}-1 / 2} \rightarrow \infty$ does not embed isomorphically as a complemented subspace into any $L_{p}, p<\infty$, so this example is not good for our purposes. Actually, at least for some sequences $\left\{\left(r_{i}, n_{i}\right)\right\}_{i}$ with the above properties, $\left(\sum_{i} \ell_{r_{i}}^{n_{i}}\right)_{2}$ does not even embed isomorphically into any $L_{p}$ space, $p<\infty$. That this is true, for example, if each $(r, n) \in\left\{\left(r_{i}, n_{i}\right)\right\}$ repeats $n$ times follows from [KS, Corollary 3.4]. 
In the next section, we show how to get complemented subspaces of the reflexive $L_{p}$ spaces that satisfy the hypotheses of Corollary 2.3.

\section{The operator $T$}

In this section we prove that, for each $1<q<2$, there is a complemented subspace of $L_{q}$ isomorphic to a space $X$ with a 1-unconditional basis on which there is an operator of norm at most 1 with properties (a) and (b) of Proposition 2.1.

Recall that, for a sequence $u=\left\{u_{j}\right\}_{j=1}^{\infty}$ of positive real numbers and for $p>2$, the Banach space $\mathfrak{X}_{p, u}$ is the sequence space with norm

$$
\left\|\left\{a_{j}\right\}_{j=1}^{\infty}\right\|=\max \left\{\left(\sum_{j=1}^{\infty}\left|a_{j}\right|^{p}\right)^{1 / p},\left(\sum_{j=1}^{\infty}\left|a_{j} u_{j}\right|^{2}\right)^{1 / 2}\right\} .
$$

Rosenthal $[\mathrm{R}]$ proved that $\mathfrak{X}_{p, u}$ is isomorphic to a complemented subspace of $L_{p}$ with the isomorphism constant and the complementation constant depending only on $p$. If $u$ is such that, for all $\varepsilon>0$,

$$
\sum_{u_{j}<\varepsilon} u_{j}^{2 p /(p-2)}=\infty
$$

then one gets a space isomorphically different from $\ell_{p}, \ell_{2}$ and $\ell_{p} \oplus \ell_{2}$. However, for different $u$ satisfying the condition above, the different $\mathfrak{X}_{p, u}$ spaces are mutually isomorphic. We denote by $\mathfrak{X}_{p}$ any of these spaces. Later, we shall need more properties of the spaces $\mathfrak{X}_{p, u}$, and of particular embeddings of them into $L_{p}$, but for now we only need the representation (3.1), and we think of $\mathfrak{X}_{p, u}$ as a subspace of $\ell_{p} \oplus_{\infty} \ell_{2}$.

Let $\left\{e_{j}\right\}_{j=1}^{\infty}$ be the unit vector basis of $\ell_{p}$, and let $\left\{f_{j}\right\}_{j=1}^{\infty}$ be the unit vector basis of $\ell_{2}$. Let $v=\left\{v_{j}\right\}_{j=1}^{\infty}$ and $w=\left\{w_{j}\right\}_{j=1}^{\infty}$ be two positive real sequences such that $\delta_{j}=$ $w_{j} / v_{j} \rightarrow 0$ as $j \rightarrow \infty$ and $\max _{j=1}^{\infty} \delta_{j} \leqslant 1$. Set

$$
g_{j}^{v}=e_{j}+v_{j} f_{j} \in \ell_{p} \oplus_{\infty} \ell_{2} \quad \text { and } \quad g_{j}^{w}=e_{j}+w_{j} f_{j} \in \ell_{p} \oplus_{\infty} \ell_{2}
$$

Then, $\left\{g_{j}^{v}\right\}_{j=1}^{\infty}$ is the unit vector basis of $\mathfrak{X}_{p, v}$ and $\left\{g_{j}^{w}\right\}_{j=1}^{\infty}$ is the unit vector basis of $\mathfrak{X}_{p, w}$. Define also

$$
\Delta: \mathfrak{X}_{p, w} \longrightarrow \mathfrak{X}_{p, v}
$$

by

$$
\Delta g_{j}^{w}=\delta_{j} g_{j}^{v}
$$

Note that $\Delta$ is the restriction to $\mathfrak{X}_{p, w}$ of $K \in L\left(\ell_{p} \oplus_{\infty} \ell_{2}\right)$ defined by

$$
K\left(e_{j}\right)=\delta_{j} e_{j} \quad \text { and } \quad K\left(f_{j}\right)=f_{j}
$$


Consequently, $\|\Delta\| \leqslant\|K\|=1$.

The following proposition follows immediately from the easily verified fact that $\left\|\left.K\right|_{\left[e_{j}\right]_{j=m}^{\infty}}\right\| \rightarrow 0$ as $m \rightarrow \infty$.

Proposition 3.1. Given n, there exists an $m$ such that, if $E$ is an n-dimensional subspace of $\left[e_{j}\right]_{j=m}^{\infty} \oplus\left[f_{j}\right]_{j=1}^{\infty} \subset \ell_{p} \oplus_{\infty} \ell_{2}$, then $\gamma_{2}\left(\left.K\right|_{E}\right) \leqslant 2$. In particular, if $E$ is an $n$ dimensional subspace of $\left[g_{j}^{w}\right]_{j=m}^{\infty} \subset \mathfrak{X}_{p, w}$, then $\gamma_{2}\left(\left.\Delta\right|_{E}\right) \leqslant 2$.

Next, we define weights $\left\{v_{j}\right\}_{j}$ and $\left\{w_{j}\right\}_{j}$, with some additional properties. For that, we use different representations of the spaces $\mathfrak{X}_{p, u}$. It was proved in $[\mathrm{R}]$ that, if $\left\{X_{j}\right\}_{j=1}^{\infty}$ is a sequence of symmetric, each 3 -valued, independent random variables all $L_{p}$-normalized, $2<p<\infty$, then $\left\{X_{j}\right\}_{j=1}^{\infty}$ is equivalent, in $L_{p}$, to $\left\{g_{j}^{u}\right\}_{j=1}^{\infty}$, the unit vector basis of $\mathfrak{X}_{p, u}$, where $u_{j}=\left\|X_{j}\right\|_{2}$. Defining $Y_{j}=X_{j} /\left\|X_{j}\right\|_{q}$, for $q=p /(p-1),\left\{Y_{j}\right\}_{j=1}^{\infty}$ is equivalent, in $L_{q}$, to the basis $\left\{h_{j}^{u}\right\}_{j=1}^{\infty}$ of $\mathfrak{X}_{q, u}:=\mathfrak{X}_{p, u}^{*}$, that is dual to the unit vector basis of $\mathfrak{X}_{p, u}$.

Let us say already at this early stage that, for some appropriate weights $\left\{v_{j}\right\}_{j}$ and $\left\{w_{j}\right\}_{j}$, the operator $T$ we are after will be of the form $\Delta^{*}$ followed by a norm-1 isomorphism from $\mathfrak{X}_{q, w}$ to $\mathfrak{X}_{q, v}$.

Recall that $P: L_{p} \rightarrow\left[X_{j}\right]_{j=1}^{\infty}$ defined by

$$
P f=\sum_{j=1}^{\infty}\left(\int_{0}^{1} f Y_{j}\right) X_{j}
$$

defines a bounded projection onto $\left[X_{j}\right]_{j=1}^{\infty}$ (and $P^{*}$ a bounded projection from $L_{q}$ onto $\left.\left[Y_{j}\right]_{j=1}^{\infty}\right)$. The norms of the equivalences above and of the projections depend on $p$, but not on the particular weights $u$.

We now recall a construction from [JS, §4]. It was shown there that, given $1<q<2$, any sequence $\left\{\delta_{i}\right\}_{i=1}^{\infty}$ that decreases to zero, any sequence $\left\{r_{i}\right\}_{i=1}^{\infty}$ such that $q<r_{i} \uparrow 2$ fast enough and in particular satisfying $\delta_{i}^{q\left(2-r_{i}\right) /(2-q)}>\frac{1}{2}, i=1,2, \ldots$, and for any sequence $\varepsilon_{i} \downarrow 0$, we can find two sequences $\left\{Y_{i}\right\}_{i}$ and $\left\{Z_{i}\right\}_{i}$ of symmetric, independent, 3-valued random variables, all normalized in $L_{q}$, with the following additional properties:

- Put $v_{j}=1 /\left\|Y_{j}\right\|_{2}$ and $w_{j}=1 /\left\|Z_{j}\right\|_{2}$. Then, there are disjoint finite subsets $\sigma_{i}$, $i=1,2, \ldots$, of the integers such that $w_{j}=\delta_{i} v_{j}$ for $j \in \sigma_{i}$.

- There are independent random variables $\left\{\bar{Y}_{i}\right\}_{i} r_{i}$-stable normalized in $L_{q},\left\{\bar{Z}_{i}\right\}_{i}$ $r_{i}$-stable with $1 \geqslant\left\|\bar{Z}_{i}\right\|_{q} \geqslant \frac{3}{4}$ for each $i$, and there are coefficients $\left\{a_{j}\right\}_{j}$ such that

$$
\left\|\bar{Y}_{i}-\sum_{j \in \sigma_{i}} a_{j} Y_{j}\right\|_{q}<\varepsilon_{i} \text { and }\left\|\bar{Z}_{i}-\sum_{j \in \sigma_{i}} \delta_{i} a_{j} Z_{j}\right\|_{q}<\varepsilon_{i} .
$$


We may of course repeat each of the triplets $\left(r_{i}, \delta_{i}, \varepsilon_{i}\right)$ as many (finitely many) times as we wish. Thus we conclude that, given any sequence $\left\{\delta_{i}\right\}_{i=1}^{\infty}$ decreasing to zero, any sequence $\left\{r_{i}\right\}_{i=1}^{\infty}$ such that $q<r_{i} \uparrow 2$ and satisfying $\delta_{i}^{q\left(2-r_{i}\right) /(2-q)}>\frac{1}{2}, i=1,2, \ldots$, any sequence of integers $n_{i}$, and any sequence $\varepsilon_{i} \downarrow 0$, we can find two sequences $\left\{Y_{i}\right\}_{i}$ and $\left\{Z_{i}\right\}_{i}$ of symmetric, independent, 3 -valued random variables, all normalized in $L_{q}$, with the following additional properties:

- Put $v_{j}=1 /\left\|Y_{j}\right\|_{2}$ and $w_{j}=1 /\left\|Z_{j}\right\|_{2}$. Then there are disjoint finite subsets $\sigma_{i, l}$, $i=1,2, \ldots$ and $l=1, \ldots n_{i}$, of the integers such that $w_{j}=\delta_{i} v_{j}$ for $j \in \sigma_{i, l}$.

- There exist independent random variables $\left\{\bar{Y}_{i, l}\right\}_{i, l} r_{i}$-stable normalized in $L_{q}$, $\left\{\bar{Z}_{i, l}\right\}_{i, l} r_{i}$-stable with $1 \geqslant\left\|\bar{Z}_{i, l}\right\|_{q} \geqslant \frac{3}{4}$ for each $i$ and $l$, and there exist coefficients $\left\{a_{j}\right\}_{j}$ such that

$$
\left\|\bar{Y}_{i, l}-\sum_{j \in \sigma_{i, l}} a_{j} Y_{j}\right\|_{q}<\varepsilon_{i} \text { and }\left\|\bar{Z}_{i, l}-\sum_{j \in \sigma_{i, l}} \delta_{i} a_{j} Z_{j}\right\|_{q}<\varepsilon_{i} .
$$

Choosing the $\varepsilon_{i}$ small enough, we may assume that $\left\{\sum_{j \in \sigma_{i, l}} a_{j} Y_{j}\right\}_{l=1}^{n_{i}}$ is, in $L_{q}$, 2-equivalent to the unit vector basis of $\ell_{r_{i}}^{n_{i}}$, and similarly $\left\{\sum_{j \in \sigma_{i, l}} \delta_{i} a_{j} Z_{j}\right\}_{l=1}^{n_{i}}$ is, in $L_{q}$, 2-equivalent to the unit vector basis of $\ell_{r_{i}}^{n_{i}}$. Denoting by $R$ the map that sends $Y_{j}$ to $\delta_{i} Z_{j}$ for $j \in \sigma_{i, l}$, we get that this map satisfies that, for all $i$, there is a space $E_{i}$ that is 2-isomorphic to $\ell_{r_{i}}^{n_{i}}$ such that $\|R x\| \geqslant \frac{1}{4}\|x\|$ for all $x \in E_{i}$. Choosing the $n_{i}$ large enough, we may also assume that, for all $k$,

$$
n_{i}^{1 / r_{i}-1 / 2} \rightarrow \infty \quad \text { as } i \rightarrow \infty
$$

Since $n_{i}^{1 / r_{i}-1 / 2}$ is the distance of $\ell_{r_{i}}^{n_{i}}$ to a Hilbert space, we get that $d\left(E_{i}\right) \rightarrow \infty$.

We are now ready to state and prove the main proposition of this section.

Proposition 3.2. With the choice of $v=\left\{v_{j}\right\}_{j}$ and $w=\left\{w_{j}\right\}_{j}$ above, set $X=\mathfrak{X}_{q, v}$, let $\Delta^{*}: \mathfrak{X}_{q, v} \rightarrow \mathfrak{X}_{q, w}$ be the adjoint of $\Delta$ defined at the beginning of this section, and let $S$ be a norm-1 isomorphism from $\mathfrak{X}_{q, w}$ onto $\mathfrak{X}_{q, v}$. Put $T=S \Delta^{*}$. Then, $X$ and $T$ satisfy the assumptions of Proposition 2.1.

Proof. Since $T=A R B$ for isomorphisms $A$ and $B$, the discussion above provides a proof of property (a). Property (b) follows by duality from Proposition 3.1. Indeed, fix $m$ and $n$ and let $E$ be an $m$-dimensional subspace of $\left[h_{i}^{v}\right]_{i \geqslant n}$. We have that $\Delta^{*}(E)$ is a subspace of $\left[h_{i}^{w}\right]_{i \geqslant n}$, so there is a $k$-dimensional subspace $F$ of $\left[g_{i}^{w}\right]_{i \geqslant n}$ that 2-norms $E$. Here, $k=k(m)$ depends only on $m$ (and we used the 1-unconditionality of the bases). By Proposition 3.1, for some $n$ depending only on $k$ and thus only on $m, \gamma_{2}\left(\left.\Delta\right|_{F}\right) \leqslant 2$. From this it is easy to get that $\gamma_{2}\left(\left.\Delta^{*}\right|_{E}\right) \leqslant 4$. Consequently, this holds also for $T=S \Delta^{*}$. 


\section{The main result and additional comments}

TheOREM 4.1. For every $1<p \neq 2<\infty$ the number of different closed ideals in $L\left(\mathfrak{X}_{p}\right)$ and in $L\left(L_{p}\right)$ is exactly $2^{2^{\aleph_{0}}}$. Moreover, each of these spaces contains an antichain of closed ideals of cardinality $2^{2^{\aleph_{0}}}$ and a chain of cardinality $2^{\aleph_{0}}$.

Proof. For $\mathfrak{X}_{q}, 1<q<2$, the theorem follows from Proposition 3.2 and Corollary 2.3. For $\mathfrak{X}_{p}, 2<p<\infty$, it follows by simple duality. Since for $1<p \neq 2<\infty$ the space $\mathfrak{X}_{p}$ is isomorphic to a complemented subspace of $L_{p}$, it follows also for $L_{p}$.

The statements about chains and antichains are a consequence of the remarks at the end of $\S 2$.

Remark 4.2. As is stated in the introduction, the new ideals in $L\left(L_{p}\right)$ and $L\left(\mathfrak{X}_{p}\right)$, $1<p \neq 2<\infty$, constructed in Theorem 4.1 are all large, and in fact contain projections whose ranges are isomorphic to $\ell_{2}$.

Proof. First, we observe that it is enough to show that, for each $\alpha \in \mathcal{C}$, the operator $T_{\alpha}$ on $X$ (recall that $X$ is isomorphic to $\mathfrak{X}_{q}$, where $1<q<2$ ), isomorphically preserves a copy of $\ell_{2}$. Here, $T$ is the operator produced in Proposition 3.2, and $T_{\alpha}$ is defined in the statement of Proposition 2.1. Indeed, since any subspace of $L_{q}, 1<q<2$, that is isomorphic to $\ell_{2}$ contains a further infinite-dimensional subspace that is complemented in $L_{q}$ (this fact was probably first observed by Pełczyński; see [JS, p. 1106] for a proof), this will show that the identity on $\ell_{2}$ factors through $T_{\alpha}$, and hence there is a projection in the ideal generated by $T_{\alpha}$ whose range is isomorphic to $\ell_{2}$. This will give Remark 4.2 for $L\left(\mathfrak{X}_{p}\right)$ when $1<p<2$, and the case of $L\left(\mathfrak{X}_{p}\right)$ for $2<p<\infty$ follows by duality. The statement for $L\left(L_{p}\right), 1<p \neq 2<\infty$, is then immediate.

To show that $T_{\alpha}$ isomorphically preserves a copy of $\ell_{2}$, note that the space $\mathfrak{X}_{q, v}$ we built contains a modular space [LT, Definition 4.d.1] $\ell_{\left\{r_{i}\right\}}$ with $r_{i} \uparrow 2$ on which $T_{\alpha}$ is an isomorphism and thus (by passing to a subsequence of the sequence $r_{i}$ that tends quickly to 2), also contains an isomorph of $\ell_{2}$ on which $T_{\alpha}$ is an isomorphism.

Remark 4.3. The large ideals in $L\left(L_{q}\right)$ and $L\left(\mathfrak{X}_{q}\right)$ constructed in Theorem 4.1 do not contain the ideal of strictly singular operators.

Sketch of proof. By $[\mathrm{W}]$ and how we constructed the ideals in $L\left(L_{q}\right)$ from the ideals in $L\left(\mathfrak{X}_{q}\right)$, it is enough to consider the ideals constructed in $L\left(\mathfrak{X}_{q}\right)$ for $1<q<2$. Let $T$ be the operator and $X$ be the space isomorphic to $\mathfrak{X}_{q}$ that are defined in Proposition 3.2 and which satisfy the assumptions of Proposition 2.1. Let $\left\{T_{\alpha}: \alpha \in \mathcal{C}\right\}$ be the corresponding operators on $X$ given by Proposition 3.2. As in the proof of Corollary 2.3, for $\mathcal{A}$ a (always non-empty, proper) subset of $\mathcal{C}$, let $\mathcal{I}_{\mathcal{A}}$ be the ideal in $L(X)$ generated by $\mathcal{A}$. 
Given $\mathcal{A} \subset \mathcal{C}$, take any $\alpha \in \mathcal{C}$ that is not in $\mathcal{A}$. We know that $T_{\alpha}$ is not in $\overline{\mathcal{I}}_{\mathcal{A}}$, but we want a strictly singular operator that is not in $\overline{\mathcal{I}}_{\mathcal{A}}$ and $T_{\alpha}$ is not strictly singular. Let $Y:=\left(\sum_{k=1}^{\infty} G_{k}\right)_{q}$, where the $G_{k}$ are the block subspaces of $X$ defined in the proof of Proposition 2.1. The $G_{k}$ are contractively complemented in $X$, and $X$ is isomorphic to a complemented subspace of $L_{q}$, hence $Y$ is isomorphic to a complemented subspace of $\ell_{q}$ (and thus to $\ell_{q}$ by Pełczyński's well-known theorem, but we do not need this), which in turn is isomorphic to a complemented subspace of $X$. Define $U: Y \rightarrow X$ by making $U$ the identity on each $G_{k}$ and extending by linearity and continuity. This is ok because $L_{q}$ has type $q$ and $\left(G_{k}\right)_{k}$ is a monotonely unconditional Schauder decomposition for a subspace of $X$, hence the decomposition $\left(G_{k}\right)$ has an upper $q$-estimate (even with constant 1 ). Let $E_{k}$ be the subspace of $G_{k}$ defined in the proof of Proposition 2.1. The operator $T_{\alpha} U$ is strictly singular and $\left\|T_{\alpha} U x\right\| \geqslant \eta\|x\|$ for all $x$ in $E_{k}$ with $k$ in $\alpha$. Since $Y$ is isomorphic to a complemented subspace of $X$, we also get a strictly singular operator $S: X \rightarrow X$ and subspaces $H_{k}$ of $Y$, with $H_{k}$ isomorphic to $E_{k}$ (with isomorphism constant independent of $k$ ), such that $\|S x\| \geqslant \eta\|x\|$ for all $x \in H_{k}$, with $k \in \alpha$. By Remark 2.2, this is enough to yield that $S$ is not in the closed ideal in $L(X)$ generated by $\left\{T_{\beta}: \beta \in \mathcal{C}\right.$ with $\left.\beta \neq \alpha\right\}$.

Remark 4.4. $L\left(L_{q}\right)$ and $L\left(\mathfrak{X}_{q}\right), 1<q \neq 2<\infty$, both contain exactly $2^{2^{\aleph_{0}}}$ closed small ideals.

Sketch of proof. Again, it is enough to deal with the case of $L\left(\mathfrak{X}_{q}\right)$ with $1<q<2$. Let $X$ and $T$ be as in Remark 4.3. For $\mathcal{A} \subset \mathcal{C}$, let $\mathcal{J}_{\mathcal{A}}$ be the ideal in $L(X)$ generated by $\left\{T_{\alpha} U P: \alpha \in \mathcal{A}\right\}$, where $P$ is any fixed projection from $X$ onto a subspace isomorphic to $Y$ (we identify $Y$ with that subspace). All $\overline{\mathcal{J}}_{\mathcal{A}}$ are small ideals, and clearly $\mathcal{J}_{\mathcal{A}}$ is contained in the ideal $I_{\mathcal{A}}$ generated by $\left\{T_{\alpha}: \alpha \in \mathcal{A}\right\}$. But in Remark 4.3 we saw that $T_{\alpha} U P$ is not contained in $\bar{I}_{\mathcal{A}}$ when $\alpha \notin \mathcal{A}$, so $\overline{\mathcal{J}}_{\mathcal{A}} \neq \overline{\mathcal{J}}_{\mathcal{B}}$ when $A \neq B$.

\section{Added in proof}

The two authors and Chris Phillips observed recently that, for any Banach space $X$, two different closed ideals in $L(X)$ are also not isomorphic as Banach algebras; i.e., are not homomorphic by an homomorphism that is continuous in both directions. It follows that there are $2^{2^{\aleph_{0}}}$ closed ideals in $L\left(L_{p}(0,1)\right), 1<p \neq 2<\infty$, that are mutually non-isomorphic as Banach algebras. 


\section{References}

[BRS] Bourgain, J., Rosenthal, H. P. \& Schechtman, G., An ordinal $L^{p}$-index for Banach spaces, with application to complemented subspaces of $L^{p}$. Ann. of Math., 114 (1981), 193-228.

[J] Johnson, W. B., Operators into $L_{p}$ which factor through $1_{p}$. J. London Math. Soc., 14 (1976), 333-339.

[JPS] Johnson, W. B., Pisier, G. \& Schechtman, G., Ideals in $L\left(L_{1}\right)$. Math. Ann., 376 (2020), 693-705.

[JS] Johnson, W. B. \& Schechtman, G., Multiplication operators on $L\left(L_{p}\right)$ and $l_{p}$-strictly singular operators. J. Eur. Math. Soc. (JEMS), 10 (2008), 1105-1119.

[KP] Kadec, M. I. \& Pęczyński, A., Bases, lacunary sequences and complemented subspaces in the spaces $L_{p}$. Studia Math., 21 (1961/62), 161-176.

[KS] Kwapień, S. A. \& Schütт, C., Some combinatorial and probabilistic inequalities and their application to Banach space theory. II. Studia Math., 95 (1989), 141-154.

[LT] Lindenstrauss, J. \& Tzafriri, L., Classical Banach Spaces. I. Ergebnisse der Mathematik und ihrer Grenzgebiete, 92. Springer, Berlin-New York, 1977.

[M] Mankiewicz, P., A superreflexive Banach space $X$ with $L(X)$ admitting a homomorphism onto the Banach algebra $C(\beta \mathbf{N})$. Israel J. Math., 65 (1989), 1-16.

[P] Pietsch, A., Operator Ideals. Mathematische Monographien, 16. VEB Deutscher Verlag der Wissenschaften, Berlin, 1978.

[R] Rosenthal, H. P., On the subspaces of $L^{p}(p>2)$ spanned by sequences of independent random variables. Israel J. Math., 8 (1970), 273-303.

[SZ] Schlumprecht, T. \& ZsÁk, A., The algebra of bounded linear operators on $\ell_{p} \oplus \ell_{q}$ has infinitely many closed ideals. J. Reine Angew. Math., 735 (2018), 225-247.

[T-J] Tomczak-Jaegermann, N., Banach-Mazur Distances and Finite-Dimensional Operator Ideals. Pitman Monographs and Surveys in Pure and Applied Mathematics, 38. Longman Scientific \& Technical, Harlow; John Wiley \& Sons, New York, 1989.

[W] WeIs, L., On perturbations of Fredholm operators in $L_{p}(\mu)$-spaces. Proc. Amer. Math. Soc., 67 (1977), 287-292.

William B. JoHnson

Department of Mathematics

Texas A\&M University

College Station, TX 77843-3368

U.S.A.

johnson@math.tamu .edu

\section{Gideon Schechtman}

Department of Mathematics

Weizmann Institute of Science

Rehovot, 76100

Israel

gideon@weizmann.ac.il

Received March 26, 2020 